\title{
Modeling of photovoltaic power uncertainties for impact analysis on generation scheduling and cost of an urban micro grid
}

\author{
Xin Wen, Dhaker Abbes, Bruno Francois* \\ L2EP, Univ. Lille, Centrale Lille, Arts et Métiers Paris Tech, HEI, EA 2697-L2EP, Laboratory of Electrical Engineering and Power \\ electronics, F-59000 Lille, France
}

Received 14 October 2019; received in revised form 15 January 2020; accepted 26 February 2020

Available online $\mathrm{xxxx}$

\begin{abstract}
In electrical systems, the main objective is to satisfy the load demand at the least cost without having imbalance between generation and consumption. Thus, the uncertainty of photovoltaic (PV) power production must be considered in generation planning of a power system. In this paper, we develop a modeling method of this uncertainty to consider it into the generation scheduling. The optimal generation scheduling in an urban microgrid is made by taking in consideration the operating reserve provision under stochastic characteristics of PV power prediction. By considering a prescribed risk level of unbalancing, a dynamic programming algorithm sets the operational planning of conventional generators by solving a non-convex mixedinteger nonlinear programming model, so that the operational cost and available operating reserve can be calculated. Then, the effect of PV power uncertainty into the unit commitment is analyzed by considering PV forecast intervals with a 95 $\%$ confidence level. The unit commitment is then recalculated with new generator set points and the same criteria. Finally, variations of the targeted minimized costs and obtained OR is analyzed according to the uncertainty.

(C) 2020 International Association for Mathematics and Computers in Simulation (IMACS). Published by Elsevier B.V. All rights reserved.
\end{abstract}

Keywords: Unit commitment; Uncertainty; Dynamic programming; Operating reserve; Micro grid

\section{Introduction}

The large-scale integration of renewable energy sources such as wind and solar generation in power systems is limited by their inner characteristics, like unpredictable primary source variations and uncontrollable power generations. These characteristics bring uncertainties in power systems, especially those microgrids that are extremely dependent upon renewable energy supplies [10,14,21]. The large-scale development of small-sized variable renewable energy sources (RES) in urban electrical systems increases local high dynamic unbalances. Thus, it can create instabilities on the inertia response and primary frequency controllers of existing conventional generators [22]. Therefore, providing an adequate operating reserve (OR) power is one solution to ensure the power system security, since it can be used to compensate the unpredictable imbalances between intermittent RES generations and consumptions [15].

Generally, selecting the conventional generating unit states (on/off) during any time step of the day is known as generation scheduling, namely as unit commitment problem (UCP) [20]. From the day ahead forecasting of the

* Corresponding author.

E-mail addresses: xin.wen@centralelille.fr (X. Wen), dhaker.abbes@yncrea.fr (D. Abbes), bruno.francois@centralelille.fr (B. Francois). 


\section{Variables}
$\delta_{m}(t)$
$s_{m}(t)$
$p_{m}(t)$
$r_{m}(t)$

\section{Parameters}

$$
\frac{p_{m}}{c_{m}^{s}}, \bar{p}_{m}
$$

\section{Time series}

$$
\begin{aligned}
& D_{t}(t) \\
& C_{t}(t) \\
& \underline{r}(t)
\end{aligned}
$$

Commitment of generator $m$ at time step $t ; \delta_{m}(t) \in\{0,1\}$.

Generator $m$ is starting up at the beginning of time step $t ; s_{m}(t) \in\{0,1\}$.

The power generation set point of generator $m$ at time step $t$.

The allocated reserve power of generator $m$ at time step $t$.

\section{Sets and indices}

$m \in \mathcal{M}$
$t \in \mathcal{T}$
$\Delta$
$p$
$r$
$\Delta, p, r \in \mathcal{F}$

\section{Functions}

$c_{m}\left(p_{m}(t)\right)$
The risk index of LOLP (Loss of Load Probability).

The minimum and maximum power generation limits of generator $m$.

The start-up penalties on operating costs.

The net demand forecast at time step $t$, the difference between the total load demand forecast and photovoltaic generation forecast.

The total conventional generation capacity of all the generators at time step $t$.

Allocated operating reserve requirements at time step $t$.

Set of conventional generators.

Set of time steps.

Feasible set of $\delta_{m}(t)$.

Feasible set of $p_{m}(t)$.

Feasible set of $r_{m}(t)$.

Set of feasible solutions.

load demand and renewable generation, UCP classically finds the optimal generation scheduling that minimizes the global operating cost. Failure to commit enough energy resources to meet expected conditions can lead to expensive actions, for example starting thermal turbines instantaneously and abruptly. However introducing uncertainties in a UCP makes conventional deterministic UC methods inadequate to solve stochastic problems, since they rarely consider renewable energy sources generation or unpredicted fluctuations of load consumption [25]. The reason is that, except for demand fluctuations, there are multiple sources of uncertainties such as faults in generator operations, deviation from wind or PV forecast, etc. Stochastic UCPs require methods to minimize the operating costs for different uncertainties or constraints, and to quantify them according to probabilities [11]. Therefore, the introduction of uncertainties in UC and energy management problem adds extra computational complexity to current methods [19].

Since UCP is a complex mix-integer programming problem, various solving methods can be used to obtain the optimal generation scheduling, e.g. priority list, dynamic programming, and Lagrangian relaxation (LR) approach [25]. Currently, the most commonly applied technique is mixed-integer linear programming (MILP). [12] presents a MILP method for day-ahead stochastic UCP incorporating solar uncertainty, taking into account reserve constraints of the microgrid systems. [24] proposes a loss of load constrained UCP by optimizing probabilistic spinning reserve corresponding to unit outage events, but uncertainties of load and RES are not considered. A MILP-based multiple time resolution UC method is presented in [2] for applications with high renewable penetration. However, if the optimization problem implies a nonlinear/ non-convex objective function or nonlinear operating constraints, then the optimal solution is difficult to achieve by applying MILP. On the contrary, a dynamic programming method has less limits regarding the convexity and linearity of the UCP. For example, [6] presented a dynamic programming algorithm to coordinate the wind and thermal generation scheduling problem for the 
operating an isolated hybrid power system. Additional physical and economic operation constraints are applied to set a compromise between system security and total production cost. Linear approximation methods can be also applied to solve the nonlinear/ nonconvex programming problem. In order to obtain costs of consumed fuel for generating a certain amount of electricity, the cost function must be approximated as a convex quadratic or piecewise linear function of the generator energy output [16]. [5] presented a mixed-integer linear formulation for the unit commitment problem of thermal units for large-scale cases, the production cost and startup cost are approximated by a piecewise linear function and a stairwise function, respectively. In this work, based on the nonlinear and nonconvex characteristics, operational cost functions are approximated as non-convex and convex quadratic functions, and a dynamic programming algorithm is chosen as the appropriate and efficient approach.

In order to secure the production/consumption balancing of a microgrid under uncertainties coming from a large renewable penetration, many works explore uncertainties model based risk assessment methods. Practical applications into account reserve allocation are discussed in [28]. Reserve is quantified with a probabilistic analysis of forecasting errors of load demand and PV generation. In [7], an optimal microgrid economic operation is applied in energy management system. Day-ahead power forecasting is based on different PV output characteristics under various weather conditions. [3] proposed a probabilistic method for generating uncertainties and is based on a point estimate method. Different renewable power uncertainty sources, like wind, solar and storage, are included in the optimal energy management of the presented microgrid. [1] formulates a MILP model that includes a conditional value for risk assessment of RES and load uncertainties when making UCP in isolated systems. However, the impact on optimal operational costs regarding reserve under PV uncertainties are not yet analyzed.

In this paper, unit commitment techniques based on dynamic programming are considered for the generation scheduling with consideration of stochastic characteristics of PV energy. The impact of PV generation uncertainties on the generation scheduling, on OR powers and on costs are analyzed. The main contributions of this work are as follows:

(1) The presented UC model includes a LOLP assessment to take into account the impact of uncertainties in an urban microgrid with high renewable penetration. The deterministic day-ahead commitment of generators and half-hourly OR powers are decided by taking into account PV generation uncertainties and load uncertainties with a prescribed risk level.

(2) A dynamic programming (DP) algorithm is carried out for the UCP to find solutions of a non-convex mixedinteger nonlinear programming (MINLP) model. Operational cost functions of conventional generators are approximated as either non-convex or convex quadratic functions, then the objective function is based on combinations of quadratic operational cost functions.

(3) The impacts of PV power uncertainty on operational costs regarding operating reserve are analyzed. By considering distributed energy resources and a 95\% confidence level, a certain forecast interval is determined with a set of quantiles for every half-hourly forecasted PV generation. The effect of the PV uncertainty is analyzed under the situation of exceed or missed PV power in each half-hour. With forecast intervals, OR can be recalculated by considering a certain level of security. Meanwhile, the generation scheduling is updated in order to respond to system demand conditions, and the impact of PV power uncertainty on the operational cost is analyzed. The maximum and the minimum costs based on the PV uncertainty are obtained.

\section{Unit commitment by dynamic programming}

\subsection{Unit commitment formulation}

UC problems search the optimal output levels of generating units over a certain period. They are running before the power delivery, generally a week up to several hours in advance, to schedule power plants for power delivery at a certain moment in the future [8].

In the presented case, micro gas turbines (MGTs) are considered here as conventional generators. The objective is to minimize the operating cost of $M$ MGTs during $T$ time steps, one day-ahead considering OR provision. $p_{m}(t)$ denotes the quantity of electricity generated by MGT $m$ at time step $t$. Here the mathematical formulation of the 
presented UC problem can be formulated as:

$$
\begin{aligned}
& \min _{\Delta, p, r} \sum_{t=1}^{T} \sum_{m=1}^{M} c_{m}\left(p_{m}(t)\right)+c_{m}^{S} s_{m}(t) \\
& \text { s.t. }(2)-(5),(\Delta, p, r) \in \mathcal{F}
\end{aligned}
$$

where $m \in \mathcal{M}$ is a set of MGTs and $t \in \mathcal{T}$ is set of time steps. $c_{m}\left(p_{m}(t)\right)$ is based on nonlinear operational cost function of MGT $m$, which indicates the fuel cost of producing $p_{m}(t)$ by using MGT $m$ during the time step $t$. $c_{m}^{S} s_{m}(t)$ is the start-up or shut-down penalties on the cost. In this study, a start-up penalty is considered equal to the consumed fuel cost during a 5 min operation at full load. Shut-down penalty is considered equal to a $2.5 \mathrm{~min}$ operation at full load [13]. $\mathcal{F}$ is the set of feasible solutions, i.e. set of feasible scheduling decision variables of commitment $\delta_{m}(t)$, power generation $p_{m}(t)$ and allocated reserve power $r_{m}(t)$ for MGT $m$ at time step $t$. Considering the characteristics of the used generators and microgrid, here other constraints such as minimum uptime and down time constraints, network constraints and losses are considered and included in $(\Delta, p, r) \in \mathcal{F}$. Binary decision variables are defined as $\delta_{m}(t)$ and refer to the state of the MGT $m$ at time step $t . s_{m}(t)=1$ refers to a MGT $m$ starts up at the beginning of a time step $t$, otherwise it equals to 0 and refers to a shutdown action:

$$
s_{m}(t)=\left\{\begin{array}{l}
\delta_{m}(t), \quad t=1, \text { initial time step } \\
\delta_{m}(t) X O R \delta_{m}(t-1), \quad t \geq 2
\end{array}\right.
$$

The produced power is constrained by a minimum power and a maximum power:

$$
\underline{p_{m}} \delta_{m}(t) \leq p_{m}(t) \leq \overline{p_{m}} \delta_{m}(t), \quad \forall m \in \mathcal{M}, \forall t \in \mathcal{T}
$$

$\underline{p_{m}}$ and $\overline{p_{m}}$ are minimum and maximum capacity limits of MGT $m$. The total produced power must be equal to the demand and the required reserve:

$$
\sum_{m=1}^{M} p_{m}(t)=D(t)+\underline{r}(t), \forall t \in \mathcal{T}
$$

The total provided reserve must be more than the required reserve:

$$
\sum_{m=1}^{M} r_{m}(t) \geq \underline{r}(t), \forall t \in \mathcal{T}
$$

A power balance between the generation power and the sum of net demand forecast and reserve power should be maintained during the operation. $D(t)$ is the net demand, namely the electricity consumption forecast minus the $\mathrm{PV}$ generation forecast at time step $t$. To deal with the uncertainties in the generation scheduling, power reserve requirements are always considered in UC constraints [18,29]. Eqs. (4) and (5) are constraints between MGT generations and reserve requirement $\underline{r}(t)$ during time step $t . r_{m}(t)$ is the reserve power from MGT $m$ at time $t$. The power reserve has a cost even when it is not used. Especially in small power systems, the cost of reserve is high. Hence it is important to have OR optimal dispatching techniques taking into consideration all unit constraints. In order to obtain reserve requirement $\underline{r}(t)$, OR quantification is demonstrated and is detailed in following Section 2.2.

In this paper, the operational cost of the gas turbine is assessed by considering partial load efficiency characteristic [13]. In order to reduce the computational costs, each operational cost exponential function $c_{m}(t)$ is fitted by a quadratic function between the maximum and minimum generation limit of each MGT. Fig. 1 shows approximate quadratic operational cost functions of three MGTs, and cost function of MGT1 is no-convex, leading to the non-convexity and nonlinear characteristics of the objective function in Eq. (1).

\subsection{Reserve power quantification}

The OR is quantified in advance (one day-ahead) according to the PV power forecasting uncertainty and load forecasting uncertainty [27]. Meanwhile, the OR is quantified by carrying out risk-constrained probabilistic techniques at each time step. Loss of Load Probability (LOLP) is the probability that a power shortage may occur. LOLP is a measure of expectation that system net demand will exceed generating capacity during a given time step. 

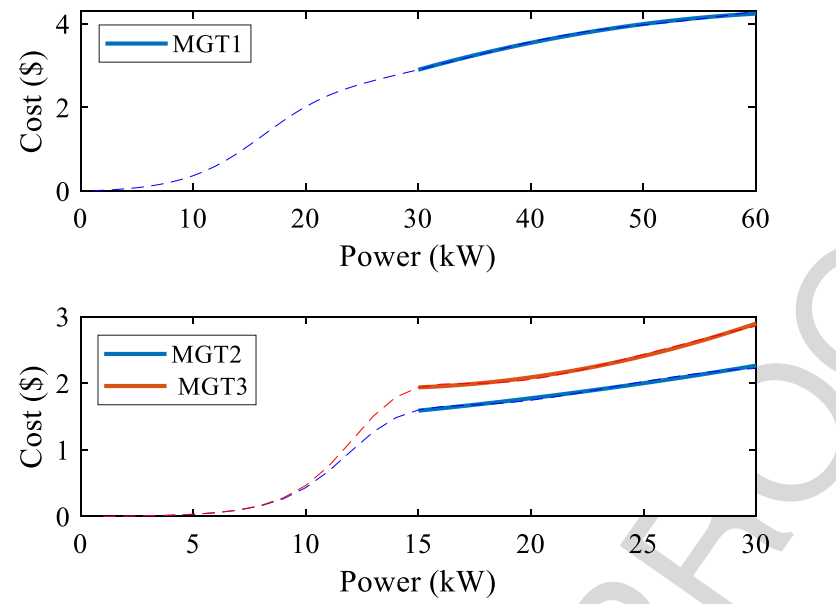

Fig. 1. The quadratic curve fitting of the cost functions of studied three MGTs.

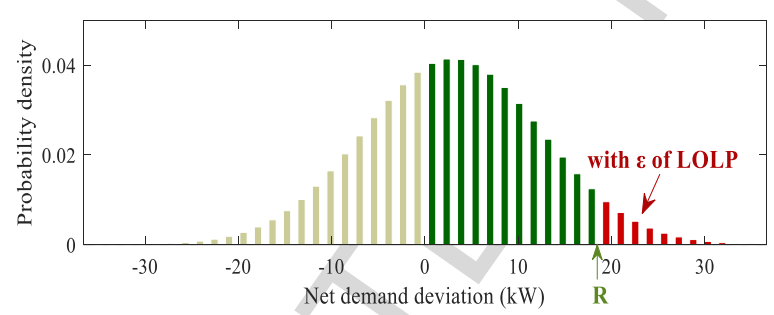

Fig. 2. Reserve calculation based on the net demand deviation $\Delta D$ with a risk index $\varepsilon$ of LOLP at ${ }_{\lambda}^{11: 00 . . ~(F o r ~ i n t e r p r e t a t i o n ~ o f ~ t h e ~}$ references to color in this figure legend, the reader is referred to the web version of this article.)

In the studied microgrid, the uncertainties coming from the PV power forecasting errors and the load forecasting errors are both taken into account:

$$
L O L P_{t}: \mathbb{P}\left[C_{t} \leq D_{t}\right], \forall t \in \mathcal{T}
$$

where $C_{t}$ is the total capacity of all MGTs at time step $t, D_{t}$ is the actual net demand at time step $t$, i.e. the difference between the real load demand and PV generation. To ensure the system reliability, constraints restricting LOLP at all time steps can be added to the UC problem, such as

$$
L O L P_{t} \leq \varepsilon
$$

where $\varepsilon$ is the risk index.

In the studied microgrid, OR power is calculated to compensate the possible power unbalance, and can be quantified according to the net demand forecasting uncertainty $p d f$ (probability density function) and $c d f$ (Cumulative distribution function) based on the probability distribution. Considering the deviation of the net demand at each time step, $L O L P_{t}$ is defined as:

$$
L O L P_{t}: 1-\mathbb{P}\left[C_{t} \geq D_{t}\right]=1-\mathbb{P}[R(t) \geq \Delta D(t)]=1-\int_{-\infty}^{R(t)} p d f(\tau) d \tau, \forall t \in \mathcal{T}
$$

$\Delta D(t)$ is the net demand deviation from the forecasted value, $R(t)$ is the reserve requirement when $L O L P_{t} \leq \varepsilon$ is satisfied. i.e. $R(t)$ is used to cover the loss of load and makes $L O L P_{t}$ be equal or less than the given risk index. Fig. 2 illustrates the calculation of reserve requirement based on the net demand deviation with a risk index $\varepsilon$ of Q4 LOLP at 11:00.

Here the presented problem is statistically discrete, since the probability distribution of net demand deviation at the same time step everyday (e.g. every day at 11:00) is discrete. The negative net demand deviation $\Delta D(t)$ (area in gray) represents the exceed generation. The positive net demand deviation represents the power deficit, i.e. the 


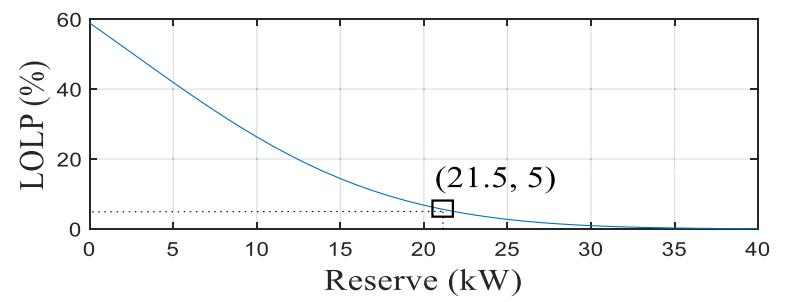

Fig. 3. LOLP curve according to reserve at 11:00.

unexpected increase in demand and deficit in PV generation capacity, so that the system load exceeds the available generating capacity. To reduce the risk of power deficit, reserve power (area in dark green) is planned according to the prescribed risk index $\varepsilon$ of LOLP (area in red). Fig. 3 presents an example of the relationship between LOLP and reserve requirement at 11:00. In the presented case, it is assumed that $\varepsilon=5 \%$, hence the $21.5 \mathrm{~kW}$ reserve is required in order to obtain a security level of $L O L P_{11: 00} \leq 5 \%$ at 11:00.

The effective OR is the difference between the sum of the maximum (or minimum) generation limits of all committed generators and the actual generation of committed generators at each time step. In this paper, efforts are made to calculate and observe both the scheduled (day-ahead) OR and effective OR scheduling based on the capacity of MGTs at each time step during the day.

\subsection{Dynamic programming mathematical formulation}

The approach of Bellman's recursive equation [4] is applied to implement an optimization procedure to solve the UCP by the formulation (1). For any MGTs state, the operational cost is expressed as the sum of:

- The cost of electricity production during the time step $[t-1, t]$ :

$$
\sum_{m=1}^{M} c_{m}\left(p_{m}(t)\right)
$$

- The cost during the previous time step $F(t-1)$ taking into account the transition cost due to the start or stop of generators:

$$
\operatorname{Tr}(t-1, t)=F(t-1)+\sum_{m=1}^{M} c_{m}^{S} s_{m}(t)
$$

At step $t$, the UCP formulation for the studied system can be expressed in the form of the recursive dynamic programming equation:

$$
F(t)=\sum_{m=1}^{M} c_{m}\left(p_{m}(t)\right)+\operatorname{Tr}(t-1, t)
$$

DP technique decomposes an optimization problem into multi-stage decision problem that can be solved successively. The DP formulation consists in 48 stages that represent the 48 time steps corresponding to each half an hour of the day after. The flow chart of the recursive DP algorithm is shown in Fig. 4.

Considering three MGTs, i.e., $M=3$ at each time step $t$ (each stage), the combinations of on/off states for all the MGTs are $2^{3}$ states. Each state is a unique combination of committed and non-committed units. Potentially feasible states are the states where demand (and reserve) can be supplied by the committed MGT units. For each potentially feasible state, the program takes all feasible states from the previous state and checks if the transition to the current state (in current time step $t$ ) is possible. If the transition is possible, calculated values are transition costs. Production for the current half-hour is calculated based on demand by taking into account production at the previous half-hour and UC constraints.

Finally, the total cost is the sum of the transition cost, production cost, and the total operating cost at the state in previous time step (stage). This procedure is repeated for all states. Total costs are then sorted and saved. If the transition to a state in current half-hour is not possible from any of the states in previous half-hour, then the current state is regarded as infeasible and is not considered anymore. 


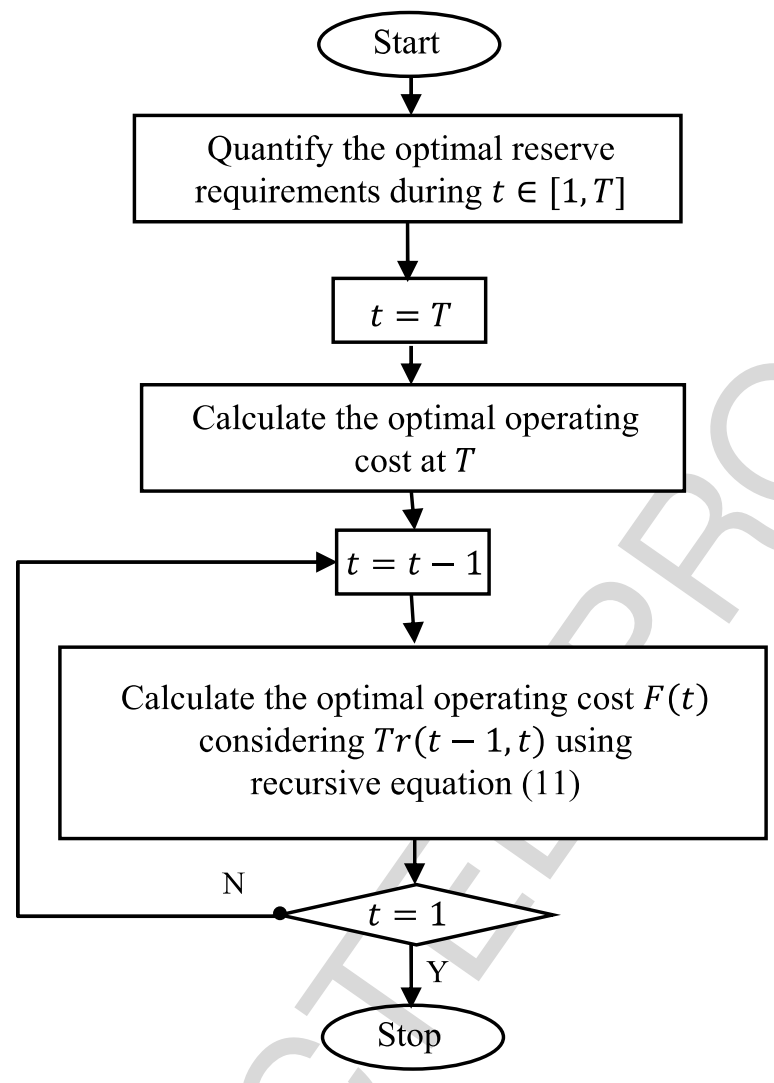

Fig. 4. The flow chart of the presented recursive DP algorithm.

\section{Dynamic programming application for generation scheduling}

\subsection{Presentation of the urban microgrid}

An urban microgrid system is considered to analyze the impact of PV power uncertainties on the operating reserve and production costs.

Owing to the stochastic characteristic of the solar energy production and load demand, predictions for both generation and consumption are essential for the optimal scheduling. In the presented urban microgrid, a dayahead approximated PV power prediction profile can be obtained each half-hour according to the meteorological information, the parameters of PV panels and the historic database of PV power. An artificial neural network is applied for the prediction [26]. Based on historic electrical power consumption, the profile of the electricity consumption can be estimated one day ahead. Methods for load forecasting are based on meteorological information and historic consumption data [17].

The studied urban microgrid includes a prosumer with $55 \mathrm{~kW}$ rated PV power, $110 \mathrm{~kW}$ residential rated load and three MGTs $(M=3)$. All power generators and electrical loads are locally connected. By comparing the daily PV energy and daily load energy, the penetration ratio of PV generation is around $25 \%$ in the studied microgrid. To simplify the study, the uncertainty from transmission line outages and voltage drops are ignored. The three MGTs have minimum and maximum power limits as follows:

$$
\begin{aligned}
& M G T 1: \underline{P_{1}}=30 \mathrm{~kW}, \overline{P_{1}}=60 \mathrm{~kW} ; \\
& M G T 2: \underline{P_{2}}=15 \mathrm{~kW}, \overline{P_{2}}=30 \mathrm{~kW} ;
\end{aligned}
$$




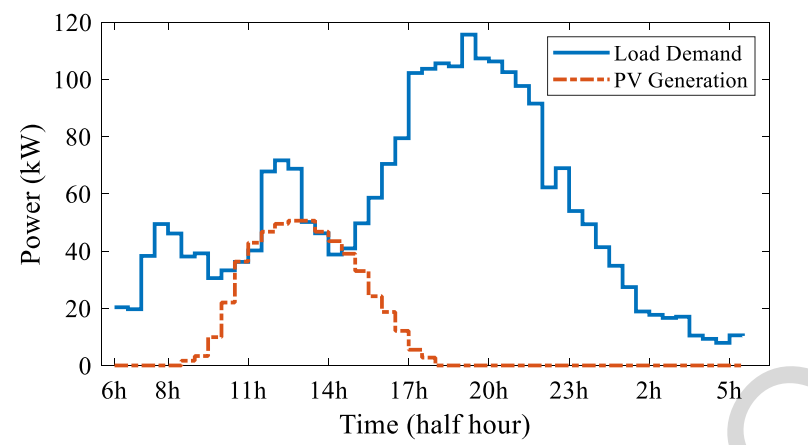

Fig. 5. Half-hourly forecasted daily PV generation, and electricity consumption.

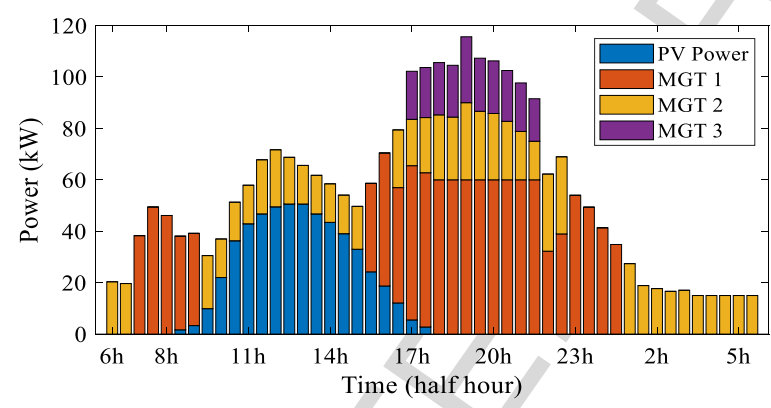

Fig. 6. Deterministic optimal day-ahead generation scheduling.

$M G T 3: \underline{P_{3}}=15 \mathrm{~kW}, \overline{P_{3}}=30 \mathrm{~kW}$.

The presented DP algorithm is implemented under Matlab R2018b on a $2.70 \mathrm{GHz}$ PC with 8 GB of installed RAM. The processing time of the optimization application is around $3.36 \mathrm{~s}$.

\subsection{Effective operating reserve with the obtained unit commitment}

The profiles of half-hourly forecasted daily PV generation and electricity consumption are given in Fig. 5. After the deterministic optimal day-ahead generation scheduling, the unit commitment result with scheduled OR provision is obtained in Fig. 6.

For each half-hour, considering generation limits of each MGT, the maximum power that can be provided by all committed MGTs is obtained, as well as the minimum power. The effective OR is the difference between the sum of the maximum (or minimum) generation limits of all committed generators and the actual generation of committed generators at each time step. The negative effective OR is obtained by the power difference between the minimum generation limits of committed MGTs and their output power at each time step. In this way, the negative OR allows the reduction of MGTs' generation under the situation of less consumption than expected, or over PV production. Similarly, the positive effective OR is provided by the difference between the maximum generation limits of committed MGTs and their output power at each time step. Both positive and negative effective OR power capacities ensure a certain level of security when there is more or less scheduled production or consumption. Obtained effective OR considering forecasted data (in Fig. 5) is shown in Fig. 7.

Moreover, half-hourly operating costs can be calculated from the fuel consumption of the MGTs for the day-ahead planned operating point and for their minimum and maximum values (Fig. 8). Generator set points vary according to varying intervals of reserve power at each time step. The maximum cost is reached if the largest amount of positive effective OR is used; while the minimum cost is obtained when all negative effective OR is consumed. 


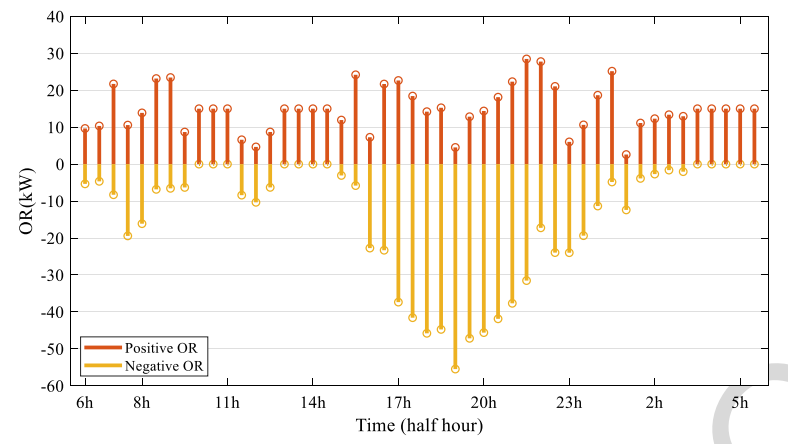

Fig. 7. Effective OR.

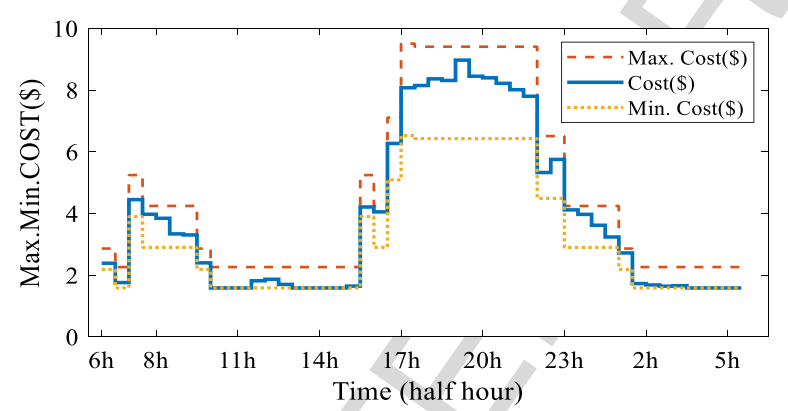

Fig. 8. Expected operating costs, maximum and minimum operating costs.

\section{Generation re-scheduling based on probabilistic forecasting}

\subsection{Characterization and definition of forecast intervals of PV generation}

Previously, the unit commitment has been calculated with the more expected PV generation forecast and by setting a 5\% risk on the net-demand unbalancing. However, in practice, PV generation and load demand are not reliable and a probabilistic level is associated to consider predicted data. In this part, the impact of PV power forecasting uncertainty on the unit commitment, generation scheduling including OR is studied.

From the past historical data, the real PV generation (in each half of an hour time step) is considered as varying around the forecasted PV power according to a probabilistic characteristic of PV forecasting errors. As a commonly used probabilistic forecasting method, quantiles/percentiles are used to characterize a certain probabilistic level of the forecasting errors [30]. The $q$ th quantile is defined as the value where the production probability less than this value is $q \%$.

The sample mean values $\overline{X^{t}}$ and sample standard deviations $S^{t}$ of forecasting errors $\left(\widehat{e^{t}}\right)$ at each time step $t$ can be calculated by:

$$
\begin{aligned}
\overline{X^{t}} & =\frac{1}{n} \sum_{i=1}^{n} \widehat{e_{i}^{t}} \\
S^{t} & =\sqrt{\frac{1}{n-1} \sum_{i=1}^{n}\left(\widehat{e_{i}^{t}}-\overline{X^{t}}\right)^{2}}
\end{aligned}
$$

Based on the given large population, the error sample data is assumed to follow a normal distribution:

$$
\widehat{e^{t}} \sim N\left(\overline{X^{t}}, \frac{\left(S^{t}\right)^{2}}{n}\right)
$$

Then, the PV power is estimated with the probabilistic characteristics of PV forecasting errors at each time step. A 3 years historical data set is available. Sample data are forecasting errors in recent 15 days on the basis of the date 

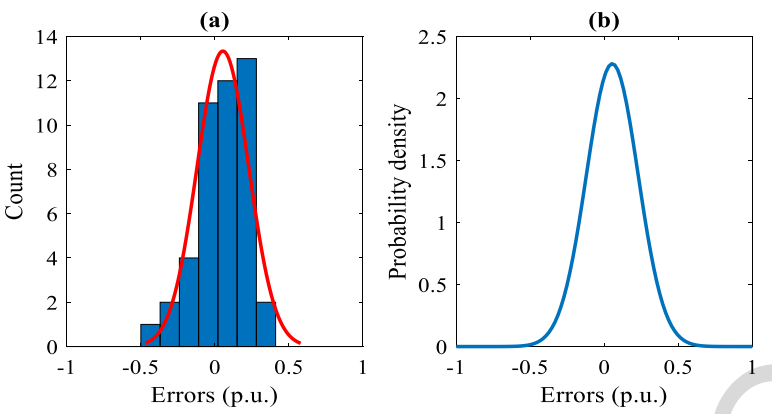

Fig. 9. (a) Frequency distribution histogram of the PV forecast errors with a normal distribution fit. (b) Normal distribution of PV forecast errors at 11:00.

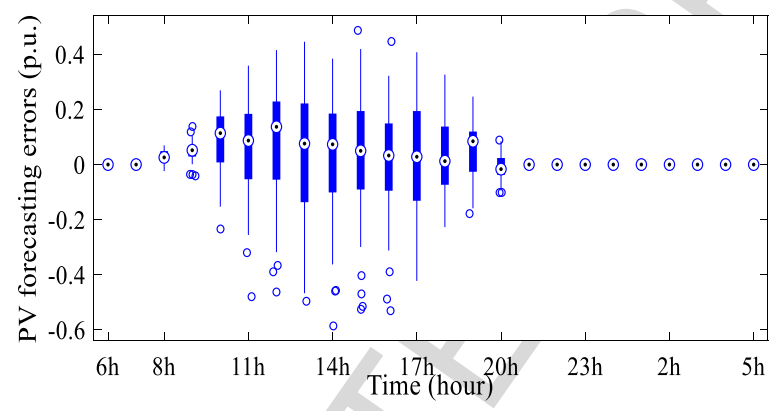

Fig. 10. The box plot of PV power forecasting errors at each time step.

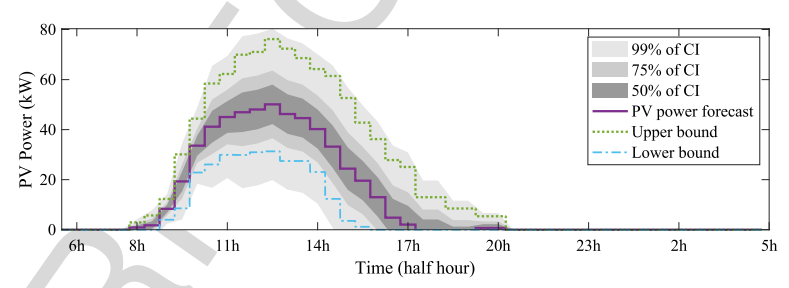

Fig. 11. Forecasted PV power according to the $95 \%$ forecast interval.

to be forecasted, i.e. data of the reference day, 7 days before the reference day and 7 days after the reference day, namely $n=45$. Different forecast errors are obtained at each time step. Fig. 9 presents the frequency histogram and probability distribution of PV forecast errors at 11:00. For each day, there are 48 sets of errors at 48 time steps if the PV forecast is done every half-hour.

In order to obtain and observe a graphical summary of PV power forecasting errors at each time step $t$, a box plot is shown in Fig. 10. Each box implies the distribution characteristics of the majority of PV forecast errors during each time step [23]. The central mark indicates the median, and the bottom and top edges of the box indicate the 25th and 75th quantiles, respectively. Their absolute values are below 0.23 per unit (p.u.).

Meanwhile, the PV power probabilistic forecasting is shown in Fig. 11. By applying a $p d f$ analysis of PV forecast errors at each time step, several probabilistic intervals are generated based on PV prediction and different security level (50\%, 75\% and 99\%). As shown in Fig. 11, a larger interval implies higher confidence level (CI) regarding the security. The 2.5th and 97.5th quantiles of the PV forecasting errors are applied here to obtain the upper bound and lower bound of the PV generation forecast. By doing so, a 95\% forecast interval is defined for each time step (Fig. 11). 


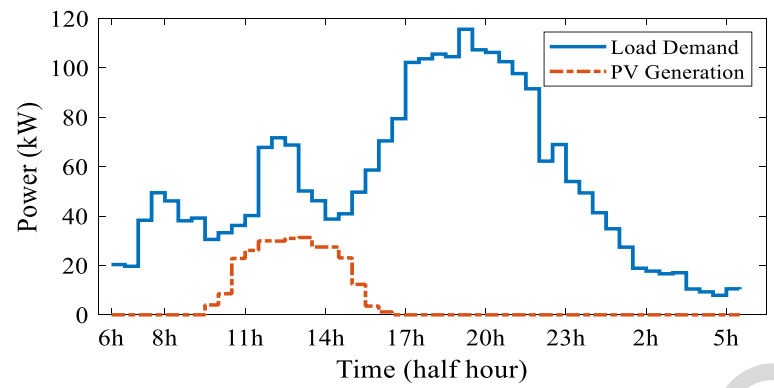

Fig. 12. Half-hourly expected lower bound of PV generation after applying forecast intervals, and daily electricity consumption.

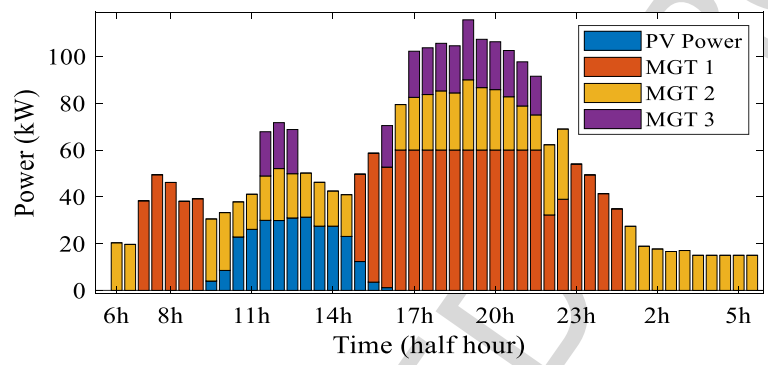

Fig. 13. Optimal dispatching of each MGT corresponding to expected lower bound of PV generation.

\subsection{Effect of $P V$ uncertainty on the effective operating reserve}

By applying the 2.5th and 97.5th quantile of PV forecasting errors at each time step, the generation re-scheduling is done according to PV variations. Considering the expected lower bound of PV generation, the most pessimistic $\mathrm{PV}$ forecast is obtained, and then a worst-case optimization is presented. The expected lower bound of half-hourly PV generation after applying forecast intervals, with daily electricity consumption are given in Fig. 12. The optimal dispatching of each MGT considering PV generation at each time step based on DP algorithm is shown in Fig. 13.

The effective OR is recalculated by taking into account power deviation of the PV generation forecast (Fig. 14). If less PV power is obtained than expected, two possibilities are considered and processed respectively:

(1) If the pre-allocated OR power is unable to handle the half-hourly PV uncertainty, then MGTs generation will be increased to provide more effective OR power to compensate the lacking PV power. For example, during time period 11:30-12:30, the reserve increases comparing to OR power with exact forecasting (Fig. 7), because one more MGT is committed (MGT 3) to provide additional positive reserves.

(2) If the pre-allocated OR power is enough to handle the half-hourly PV uncertainty, then the effective OR is consumed to compensate the PV deficit, e.g. effective OR decreases at $13 \mathrm{~h}-14 \mathrm{~h}$ comparing to Fig. 7, because positive OR is consumed to compensate the pessimistic PV.

Meanwhile, operating costs fluctuate according to generation and reserve scheduling, as shown in Fig. 15. For example at 11:00, operating cost stays the same as expected because the scheduling of the MGTs is unchanged. On the other hand, the operating cost increases at 11:30 when the scheduling is changed and there is an additional MGT turned on. Still there are variation intervals between the maximum costs (by providing the largest amount of positive OR power) and the minimum costs (by providing all of negative OR power) during each time step.

Reserves and daily operating costs under PV uncertainties are shown in Table 1. When the uncertainties of PV forecast are not considered (results are marked in gray). Expected reserves and daily costs are obtained based on the deterministic generation scheduling.

Furthermore, the results for both upper bound and lower bound of PV generation are presented respectively. When the PV uncertainties are considered according to the forecast intervals, the expected operating cost of deterministic generation scheduling is no longer able to be achieved, instead, the variation intervals of maximum and minimum costs are re-calculated. The maximum cost is obtained when considering lower bound of PV generation, 


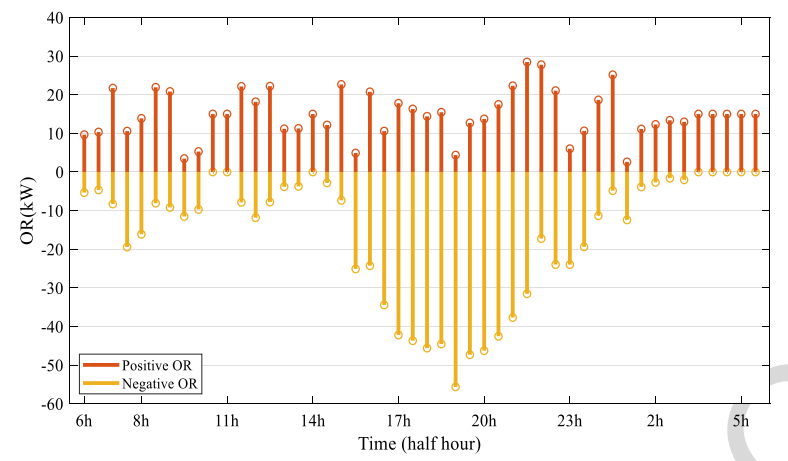

Fig. 14. Effective OR corresponding to the expected lower bound of PV generation.

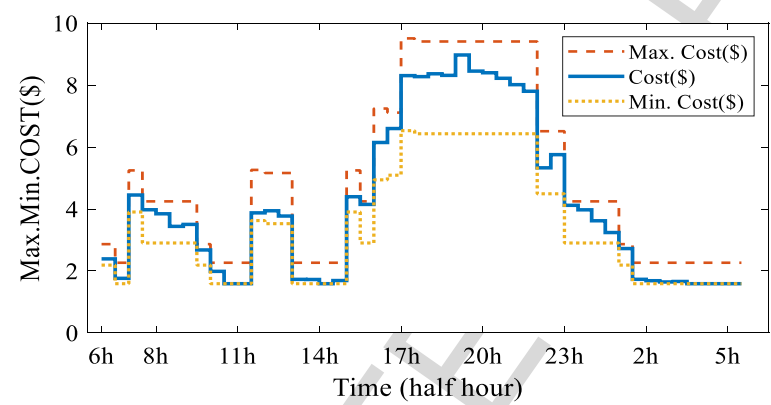

Fig. 15. Expected operating costs, maximum and minimum operating costs considering expected lower bound of PV generation.

Table 1

Reserves and daily operating costs under PV uncertainties.

\begin{tabular}{|c|c|c|c|c|c|}
\hline \multirow{2}{*}{$\begin{array}{l}\text { Net demand } \\
(\mathrm{kWh})\end{array}$} & \multirow[t]{2}{*}{$\mathrm{PV}(\mathrm{kWh})$} & \multicolumn{2}{|c|}{ Reserve $(\mathrm{kWh})$} & \multicolumn{2}{|c|}{ Daily Cost (\$) } \\
\hline & & Positive OR & $\overline{\text { Negative OR }}$ & Max. Cost & Min. Cost \\
\hline 1185 & 139 & 489 & 391 & 233 & 167 \\
\hline 1066 & 258 & 370 & 353 & 219 & 152 \\
\hline 855 & 469 & 188 & 288 & 208 & 144 \\
\hline
\end{tabular}

while minimum cost is attained when PV generation reaches upper bound of forecast interval. Meanwhile OR are re-calculated after the generation re-scheduling.

\section{Conclusions}

Following the presented OR provision method, and a prescribed risk level for both load and PV power uncertainties, this paper presents the optimization scheme of a generation scheduling for an urban microgrid with consideration of distributed energy resources and a 95\% confidence level. Furthermore, the impact of PV power uncertainty on the cost is analyzed, the maximum and the minimum costs based on the PV uncertainty are obtained. Firstly, based on nonlinear operating cost functions of generators, the presented UCP is solved by applying DP algorithm based on the established non-convex MILP model. DP algorithm calculates the generation scheduling by considering PV and load forecasting and a 5\% risk level. The scheduled (day-ahead) OR and effective OR based on the capacity of MGTs at each time step during the day are calculated.

Then, the internal uncertainty in the predicted PV generation is analyzed with a probabilistic analysis and is modeled with a pdf. According to bounds of forecast intervals, a re-scheduling if the generation planning enables the operating cost calculation for various probabilistic values of the PV power prediction. 
Further works will be oriented to integrate directly the uncertainty inside the optimization algorithm in order to obtain a stochastic analysis of predicted operating costs. Application of storage to erase uncertainty could be also considered [9].

\section{Acknowledgments}

This work is financially supported by Ecole Centrale de Lyon and China Scholarship Council (CSC).

\section{References}

[1] M. Asensio, J. Contreras, Stochastic unit commitment in isolated systems with renewable penetration under CVaR assessment, IEEE Trans. Smart Grid 7 (3) (2016) 1356-1367.

[2] E.A. Bakirtzis, P.N. Biskas, D.P. Labridis, A.G. Bakirtzis, Multiple time resolution unit commitment for short-term operations scheduling under high renewable penetration, IEEE Trans. Power Syst. 29 (1) (2014) 149-159.

[3] A. Baziar, A. Kavousi-Fard, Considering uncertainty in the optimal energy management of renewable micro-grids including storage devices, Renew. Energy 59 (2013) 158-166, http://dx.doi.org/10.1016/J.RENENE.2013.03.026.

[4] R. Bellman, Dynamic Programming, Courier Corporation, 2013.

[5] M. Carrión, J.M. Arroyo, A computationally efficient mixed-integer linear formulation for the thermal unit commitment problem, IEEE Trans. Power Syst. 21 (3) (2006) 1371-1378, http://dx.doi.org/10.1109/TPWRS.2006.876672.

[6] C.L. Chen, Optimal wind-thermal generating unit commitment, IEEE Trans. Energy Convers. 23 (1) (2008) 273-280, http://dx.doi.org/ 10.1109/TEC.2007.914188.

[7] C. Chen, S. Duan, T. Cai, B. Liu, H. G., Smart energy management system for optimal microgrid economic operation, IET Renew. Power Gener. 5 (3) (2011) 258-267, http://dx.doi.org/10.1049/iet-rpg.2010.0052.

[8] K. De Vos, Sizing and Allocation of Operating Reserves Following Wind Power Integration, KU Leuven, 2013.

[9] G. Delille, B. Francois, G. Malarange, J.L. Fraisse, Energy storage systems in distribution grids: New assets to upgrade distribution network abilities, in: CIRED 2009-20th International Conference and Exhibition on Electricity Distribution, IET.

[10] O. Erdinc, N.G. Paterakis, J.P.S. Catalão, Overview of insular power systems under increasing penetration of renewable energy sources: opportunities and challenges, Renew. Sustain. Energy Rev. 52 (2015) 333-346.

[11] M. Håberg, Fundamentals and recent developments in stochastic unit commitment, Int. J. Electr. Power Energy Syst. 109 (2019) 38-48, http://dx.doi.org/10.1016/J.IJEPES.2019.01.037.

[12] R.B. Hytowitz, K.W. Hedman, Managing solar uncertainty in microgrid systems with stochastic unit commitment, Electr. Power Syst. Res. 119 (2015) 111-118, http://dx.doi.org/10.1016/j.epsr.2014.08.020.

[13] H. Kanchev, F. Colas, V. Lazarov, B. Francois, Emission reduction and economical optimization of an urban microgrid operation including dispatched PV-based active generators, IEEE Trans. Sustain. Energy 5 (4) (2014) 1397-1405.

[14] R. Lasseter, A. Akhil, C. Marnay, J. Stephens, J. Dagle, R. Guttromson, A.S. Meliopoulous, R. Yinger, J. Eto, Integration of distributed energy resources, in: The CERTS Microgrid Concept, 2002.

[15] M.A. Matos, R.J. Bessa, Setting the operating reserve using probabilistic wind power forecasts, IEEE Trans. Power Syst. 26 (2) (2011) 594-603.

[16] J.M. Morales, A.J. Conejo, H. Madsen, P. Pinson, M. Zugno, Integrating Renewables in Electricity Markets - Operational Problems, Springer, Boston, MA, 2014.

[17] RTE (Réseau de transport d'électricité) de France. Available at: http://www.rte-france.com.

[18] P.A. Ruiz, C.R. Philbrick, E. Zak, K.W. Cheung, P.W. Sauer, Uncertainty management in the unit commitment problem, IEEE Trans. Power Syst. 24 (2) (2009) 642-651.

[19] N.V. Sahinidis, Optimization under uncertainty: state-of-the-art and opportunities, Comput. Chem. Eng. 28 (6-7) (2004) 971-983, http://dx.doi.org/10.1016/j.compchemeng.2003.09.017.

[20] S. Sen, D.P. Kothari, Optimal thermal generating unit commitment: a review, Int. J. Electr. Power Energy Syst. 20 (7) (1998) $443-451$.

[21] W. Su, J. Wang, Energy management systems in microgrid operations, Electr. J. 25 (8) (2012) 45-60, http://dx.doi.org/10.1016/J.TEJ 2012.09.010.

[22] W. Su, J. Wang, J. Roh, Stochastic energy scheduling in microgrids with intermittent renewable energy resources, IEEE Trans. Smart Grid 5 (4) (2014) 1876-1883.

[23] J.W. Tukey, Box-and-Whisker Plots, §2C in Exploratory Data Analysis, vol. 2, Addison-Wesley, Reading, MA, 1977.

[24] M.Q. Wang, M. Yang, Y. Liu, X.S. Han, Q. Wu, Optimizing probabilistic spinning reserve by an umbrella contingencies constrained unit commitment, Int. J. Electr. Power Energy Syst. 109 (2019) 187-197, http://dx.doi.org/10.1016/J.IJEPES.2019.01.034.

[25] A.J. Wood, B.F. Wollenberg, G.B. Sheblé, Power Generation, Operation, and Control, John Wiley \& Sons, 2013.

[26] X. Yan, D. Abbes, B. Francois, Solar radiation forecasting using artificial neural network for local power reserve, in: International Conference on Electrical Sciences and Technologies in Maghreb (CISTEM), Tunis, 3-6 Novembre, 2014, pp. 1-6.

[27] X. Yan, D. Abbes, B. Francois, Uncertainty analysis for day ahead power reserve quantification in an urban microgrid including PV generators, Renew. Energy 106 (2017) 288-297.

[28] X. Yan, D. Abbes, B. Francois, Development of a tool for urban microgrid optimal energy planning and management, Simul. Model. Pract. Theory 89 (2018) 64-81.

[29] X. Yan, X. Wen, B. Francois, D. Abbes, Management and dispatching of distributed operating power reserve in an urban microgrid beyond DSO risk decision, in: CIRED Workshop, Ljubljana, 2018.

[30] Q.P. Zheng, J. Wang, A.L. Liu, Stochastic optimization for unit commitment-a review, IEEE Trans. Power Syst. 30 (4) (2015) 1913-1924. 